\title{
Tumor suppressor candidate 3: A novel grading tool and predictor of clinical malignancy in human gliomas
}

\author{
JING YUAN ${ }^{1,2^{*}}$, XINSHUANG YU ${ }^{3 *}$, AIHUA WANG ${ }^{2 *}$, YAN LI $^{1 *}$, FENGJUN LIU $^{3}$, \\ YAO WANG ${ }^{1}$, SHANMEI SUN ${ }^{4}$, XIUYANG BING ${ }^{4}$, YIMING LIU ${ }^{5}$ and JUAN DU ${ }^{1,2}$ \\ ${ }^{1}$ Medical Research Center; Departments of ${ }^{2}$ Neurology, ${ }^{3}$ Radiation Oncology and \\ ${ }^{4}$ Traditional Chinese Medicine, Shandong Provincial Qianfoshan Hospital, Shandong University, Jinan, Shandong 250014; \\ ${ }^{5}$ Department of Neurology, Qilu Hospital of Shandong University, Jinan, Shandong 250012, P.R. China
}

Received June 14, 2017; Accepted November 21, 2017

DOI: $10.3892 / \mathrm{ol} .2018 .8082$

\begin{abstract}
For several years, the cause of autosomal recessive mental retardation has been attributed to the deletion or mutation of a gene named tumor suppressor candidate 3 (TUSC3). Previous research has identified that TUSC 3 is a potential tumor suppressor gene in oral epidermoid carcinoma, lung cancer and esophageal cancer. However, to the best of our knowledge, no previously published data has existed on the expression of TUSC 3 in gliomas. The present study focused on the expression of TUSC 3 in brain gliomas. Additionally, the present study sought to identify he association between TUSC 3 expression and the typical clinical and pathological disease manifestations of gliomas. TUSC3 levels were evaluated using a western blot assay and immunohistochemistry on tissue microarray slides. Results indicated a significant decrease in TUSC3 expression in glioma tissues compared with the normal adjacent tissues. Furthermore, TUSC 3 expression and World Health Organization grade demonstrated an inverse association in patients with glioma. This revealed that lower levels of TUSC3 in gliomas may be associated with a poorly-differentiated (high grade) tumor and thus a higher malignancy. Through the combination of the results of the present study and future research projects, TUSC3 may be a novel grading tool that assists with evaluating tumor malignancy and consequently a more active therapeutic regimen may be used in patients with glioma.
\end{abstract}

Correspondence to: Professor Yiming Liu, Department of Neurology, Qilu Hospital of Shandong University, 107 Wenhua xi Road, Jinan, Shandong 250012, P.R. China

E-mail: amyliu@sdu.edu.cn

Dr Juan Du, Medical Research Center, Shandong Provincial Qianfoshan Hospital, Shandong University, 16766 Jingshi Road, Jinan, Shandong 250014, P.R. China

E-mail: sunnydujuan@aliyun.com

*Contributed equally

Key words: tumor suppressor candidate 3, gliomas, World Health Organization grade

\section{Introduction}

The most prevalent primary malignancy in the central nervous system is the glioma, which constitutes $\sim 77 \%$ of all malignant brain tumors worldwide in $2007(1,2)$. At present, the pathogenesis of gliomas remains unknown. Nomenclature of gliomas is specific to the type of cell affected, including astrocytomas, oligodendrogliomas, ependymomas and mixed glioma (1-3). The glioma shares histological features with each specific cell type, but does not necessarily originate from the cell type depicted in its nomenclature (4). A variety of grading systems for tumor types are available, but the World Health Organization (WHO) grading system (2) is the most common for gliomas. This system categorizes tumor types from grade I to grade IV, with grade I being the least advanced and having the most positive prognosis to IV being the most advanced and having the worst prognosis.

The conventional therapeutic regimen for glioma consists of varying combinations of surgical procedures, radiotherapy and chemotherapy. However, this approach has been ineffective for a number of patients, resulting in a rapid progression of the disease (5). For refractory forms of the disease, previous studies have focused on molecular biomarkers to identify novel drug targets, resulting in the development of novel therapeutic approaches, which demonstrate encouraging results (6).

The tumor suppressor candidate 3 (TUSC3) gene, also referred to as N33, M33, MRT7, MRT22, oligosaccharyltransferase (OST) 3 homolog A (OST3) and D8S1992, is positioned on chromosome region 8p22 and encodes the $34 \mathrm{kDa}$ TUSC 3 protein (7). It was originally discovered by MacGrogan et al (8) in 1996. The TUSC3 mRNA may be present in large quantities in a variety of cells and tissues including prostate, colon, lung, liver, ovary, placenta, testis, brain and adipose tissues (9), yet it is rare in other tissues, including in bone marrow. TUSC3 shares a substantial amount of homologous sequences with Ost3p, a subunit of the OST complex, and serves a function in the $\mathrm{N}$-linked glycosylation reactions of the protein folding process (10-12). With emerging research conducted over the past several years, it has been determined that TUSC 3 is associated with autosomal recessive mental retardation (ARMR) (13-15). Previously, studies had identified the deletion of TUSC 3 in a number of cancer types 
including oral squamous cell carcinoma and lung cancer. This resulted in the identification of TUSC3 as a potential tumor suppressor gene (16-17). Multiple studies have been conducted to determine the association between TUSC3 expression and the development of a tumor (16-20). However, to the best of our knowledge, there has been no previously published data on the expression of TUSC 3 in gliomas. In the present study, an investigation surrounding the expression of TUSC3 in brain glioma was conducted and the association between TUSC3 expression and the clinicopathological parameters of gliomas was determined. The results of the present study may help to identify the potential prognostic and therapeutic targets for brain gliomas.

\section{Materials and methods}

Tissue samples. A total of 12 pairs of glioma tissue samples were surgically removed from patients at the Qianfoshan Hospital (Shandong, China). A total of 6 male and 6 female patients participated in the study, and the mean age was 53.1 years (range, 34-73). The tissues were obtained between August 2015 and October 2015. None of the patients had been exposed to any other therapeutic interventions, including chemotherapy or radiotherapy, prior to this surgery. Tissue microarray slides were purchased from Alenabio (Xi'an, China). The slides included 38 cases of astrocytoma, 14 cases of glioblastoma, 6 cases of oligodendrogliomas, 1 case of anaplastic ependymoma, 1 case of medulloblastoma and 13 cases of normal adjacent tissue. Patients providing adequate histological material and complete clinical data were eligible to participate in the present study. Patients with a history of chemotherapy, radiotherapy or other therapeutic interventions which may affect the results of the present study were excluded. Table I represents the clinicopathological characteristics of patients with brain glioma, who were clinically staged according to the WHO grading system (2). The WHO grading scale is as follows: WHO grade I are well-differentiated, WHO grade II are moderately-differentiated, WHO grade III are poorly differentiated and WHO grade IV are undifferentiated. WHO grade I-II are referred to as low-grade gliomas and WHO grade III-IV are referred to as high-grade gliomas. The clinicopathological information of all participants are provided in Table I. Tumor classification of each of the patients was determined by two pathologists from Shandong Provincial Qianfoshan Hospital (Shandong, China) through use of the WHO grading system. The Ethical Committee of Shandong Provincial Qianfoshan Hospital ethically approved present study, and all patients prior to the study provided written informed consent.

Western blot analysis. Tissue samples were homogenized using Radio Immunoprecipitation Assay buffer (Beyotime Institute of Biotechnology, Haimen, China) with proteinase inhibitors, resulting in cell lysis. The proteins were quantified by BCA protein quantitative kit (Beyotime Institute of Biotechnology, Haimen, China) SDS-PAGE (12\%) was administered to equal amounts $(30 \mu \mathrm{g})$ protein from each lysate and the resulting products were transferred onto a polyvinylidene membrane. Blocking of non-specific binding was achieved by placing the membrane in 5\% non-fat dried milk containing $0.1 \%$ Tween-20 at room temperature for $3 \mathrm{~h}$. The membrane was incubated at $4^{\circ} \mathrm{C}$ overnight in the presence of primary antibodies for human TUSC3 goat polyclonal antibody (dilution, 1:400; cat no. sc-98191; Santa Cruz Biotechnology, Inc., Dallas, TX, USA) and $\beta$-actin (dilution, 1:1,000; cat no. sc-98191; Santa Cruz Biotechnology, Inc., Dallas, TX, USA). Subsequent to 3 washes with PBST buffer (PBS with $0.05 \%$ Tween-20) to remove unbound primary antibodies, the membrane was further incubated for $1 \mathrm{~h}$ at room temperature with horseradish peroxidase (HRP)-conjugated secondary antibodies (rabbit anti-goat IgG, dilution, 1:5,000; cat no. TA130031; OriGene Technologies, Inc., Beijing, China). Following washing, the protein was identified through detection of immunoreactive bands using Western Lightning Chemiluminescence Reagent (Millipore; Merck KGaA, Darmstadt, Germany) and the bands were analyzed using ImageJ (version 1.5; National Institutes of Health, Bethesda, MD, USA).

Immunohistochemistry (IHC) assay. IHC staining was performed directly on the $5-\mu \mathrm{m}$ tissue slides. The first step was to dewax the slides using xylene. Following wax removal, the slides were rehydrated using a series of decreasing alcohol concentrations $(100,90,70$ and $50 \%$ ethanol; 5 min each) followed by incubation for $2 \mathrm{~h}$ at $56^{\circ} \mathrm{C}$. Then, $3 \% \mathrm{H}_{2} \mathrm{O}_{2}$ was administered for $\sim 20 \mathrm{mins}$ at room temperature to block endogenous peroxidase activity. For antigen retrieval, sodium citrate $(0.01 \mathrm{M}, \mathrm{pH} 6.0)$ was used as a buffer. The slides were incubated at $95^{\circ} \mathrm{C}$ for $20 \mathrm{~min}$ in a household microwave oven $(600 \mathrm{~W})$. Next, $10 \%$ normal goat serum (cat no. ZLI-9022; OriGene Technologies, Inc., Rockville, MD, USA) was added to the slides to block non-specific binding sites in order to reduce background staining. The slides were then incubated with the TUSC3 goat polyclonal antibody (dilution, 1:100; cat no. sc-98191; Santa Cruz Biotechnology, Inc.) overnight at $4^{\circ} \mathrm{C}$. Phosphate-buffered saline was used to wash the slides 3 times prior to application of the bio-labeled secondary antibody, Poly-HRP anti-goat immunoglobulin G secondary antibody (cat no. PV-9003; OriGene Technologies, Inc.) at a $1: 200$ dilution for $40 \mathrm{~min}$ at $37^{\circ} \mathrm{C}$. The sections were stained with diaminobenzidine at room temperature for $2 \mathrm{~min}$, followed by a counterstain with hematoxylin at room temperature for $1 \mathrm{~min}$. The slides were dehydrated using increasing concentrations of alcohol and mounted using neutral gum. Photomicrographs were captured under a light microscope (magnification, x400; BX-51, Olympus Corporation, Tokyo, Japan). Aperio Image Scope (version 12.0.1.5027; Aperio Technologies, Inc., Vista, CA, USA) was used to score the stained cells using a digital pathology system.

The cytoplasm and cell membrane were identified as regions of TUSC3 expression. Two pathologists from the Shandong Provincial Qianfoshan Hospital independently evaluated the immunoreactivity of TUSC3 expression, then groups were determined according to the respective immunoreactive score (IRS). IRS are calculated by multiplying the staining intensity by the percentage of positively stained cells. Staining intensity was classified as 0 (negative), 1 (weak), 2 (moderate) or 3 (strong). Percentage of positively stained cells was scored as 0 (negative), 1 ( $<25 \%$ of the cells), 
Table I. Baseline clinicopathological characteristics of patients with glioma and patients providing control tissues.

\begin{tabular}{|c|c|c|c|c|c|}
\hline \multirow[b]{2}{*}{ Characteristics } & \multirow[b]{2}{*}{ Number } & \multicolumn{2}{|c|}{ TUSC 3 expression } & \multirow[b]{2}{*}{ Positive rate (\%) } & \multirow[b]{2}{*}{ P-value ${ }^{\mathrm{a}}$} \\
\hline & & Positive & Negative & & \\
\hline \multicolumn{6}{|l|}{ Control patients } \\
\hline Age median (range), years & $46(26-76)$ & & & & \\
\hline \multicolumn{6}{|l|}{ Gender $(\%)$} \\
\hline Male & $6(46.2)$ & & & & \\
\hline Female & $7(53.8)$ & & & & \\
\hline \multicolumn{6}{|l|}{ Patients with glioma } \\
\hline Age median (range), years & $43(14-66)$ & & & & 0.007 \\
\hline$<43$ & $30(50.0)$ & 6 & 24 & 20.0 & \\
\hline$\geq 43$ & $30(50.0)$ & 17 & 13 & 56.7 & \\
\hline Gender & & & & & 1.000 \\
\hline Male & $35(58.3)$ & 12 & 23 & 34.3 & \\
\hline Female & $25(41.7)$ & 8 & 17 & 32.0 & \\
\hline \multicolumn{6}{|l|}{ Pathological classification } \\
\hline Astrocytoma & $38(63.3)$ & 18 & 20 & 47.4 & \\
\hline Oligodendroglioma & $6(10.0)$ & 5 & 1 & 83.3 & \\
\hline Glioblastoma & $14(23.4)$ & 0 & 14 & 0 & \\
\hline Other types & $2(3.3)$ & 0 & 2 & 0 & \\
\hline \multicolumn{6}{|l|}{ WHO grade gr $^{b}$} \\
\hline I & $1(1.7)$ & 1 & 0 & 100 & \\
\hline II & $36(60.0)$ & 20 & 16 & 55.6 & \\
\hline III & $9(15.0)$ & 3 & 6 & 33.3 & \\
\hline IV & $14(23.3)$ & 0 & 14 & 0 & \\
\hline
\end{tabular}

a Positive rates of TUSC 3 expression were compared by Fisher's exact test; ${ }^{\text {b}} \mathrm{WHO}$ grade was evaluated by two 2 independent pathologists from Qianfoshan Hospital (Jinan, China). WHO grade I-II (low-grade) gliomas are well-differentiated, whereas WHO grade III-IV (high-grade) gliomas are undifferentiated or anaplastic. TUSC, tumor suppressor candidate; WHO, World Health Organization.

2 (25-50\% of the cells), 3 (50-75\% of the cells) or 4 (>75\% of the cells). Through multiplication of these two scores, a final score was calculated. An IRS value of 6 was determined to be the cut-off value separating specimens into two groups of negative or positive expression.

Statistical analysis. Statistical analysis of the results was conducted using SPSS 13.0 software (SPSS, Inc., Chicago, IL, USA). Differences in the grayscale value of protein expression were compared using a paired-t test. The data are presented as the mean \pm standard error. Differences in TUSC3 expression between two groups were compared using a Wilcoxon's rank sum test. The Spearman's rank correlation coefficient method was used to determine the strength and direction of the association between two groups. A Fisher's exact test was performed to compare the positive rate between two groups. The comparison between clinicopathological parameters (age, gender, histological classifications and WHO grading) were performed using a Fisher's exact test. All reported P-values were two-sided and $\mathrm{P}<0.05$ was considered to indicate a statistically significant difference. A receiver operating characteristic curve was used to determine the cut-off IRS value of 6 for TUSC3 expression levels in patients. Relative TUSC3 expression for each band was analyzed using ImageJ (version 1.51; National Institutes of Health, Bethesda, MD, USA).

\section{Results}

Comparison of TUSC3 protein expression between tumor and normal adjacent brain tissue in patients with glioma. To begin with, the TUSC3 expression levels were determined through western blot analysis in glioma tissues and normal adjacent tissues, which were surgically collected at Qianfoshan Hospital. TUSC3 expression was significantly reduced in glioma tissues when compared with normal adjacent tissue $(1.631 \pm 0.4534$ vs. $0.5579 \pm 0.1723 ; \mathrm{P}=0.038$; Fig. 1). These results are indicative of the pivotal function TUSC3 may serve in glioma development.

Basic clinicalfeature of patients for tissue array. Furthermore, the levels of TUSC3 expression were evaluated using a tissue array. Table I outlines the basic clinicopathological features of the patients with glioma and control patients who provided normal adjacent tissues. The median age of the controls was 46 years (range, 26-76 years) and that of patients with glioma was 43 years (range, 14-66 years). Difference in sex 
Table II. Comparison of TUSC3 expression between normal tissue and glioma tissue.

\begin{tabular}{|c|c|c|c|c|c|}
\hline \multirow[b]{2}{*}{ Characteristics } & \multirow[b]{2}{*}{ Number } & \multicolumn{2}{|c|}{ TUSC 3 expression } & \multirow[b]{2}{*}{ Positive rate (\%) } & \multirow[b]{2}{*}{ P-value } \\
\hline & & Positive & Negative & & \\
\hline Normal adjacent tissue & 13 & 10 & 3 & 76.9 & 0.029 \\
\hline Glioma tissue & 60 & 24 & 36 & 40.0 & \\
\hline
\end{tabular}

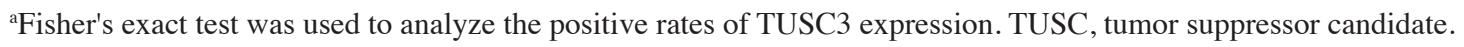

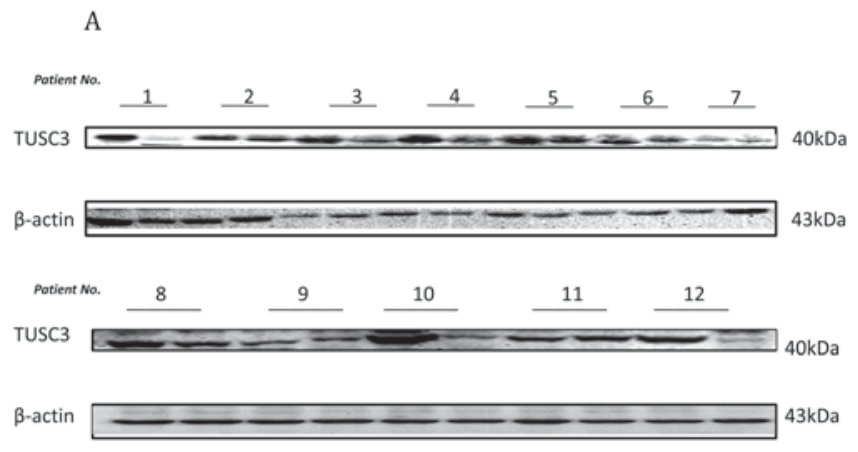

B

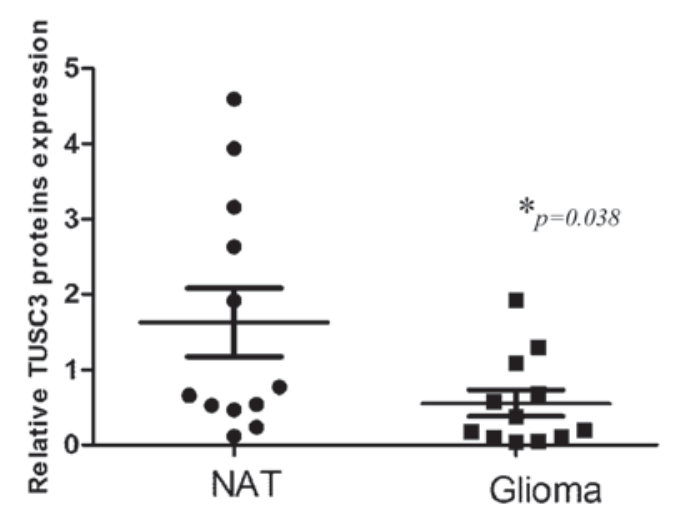

Figure 1. TUSC3 expression was detected in glioma tissues and NAT. (A) TUSC 3 expression was detected in glioma tissues and NAT by western blot analysis. Each number represented one patient (left lane: NAT and right lane: glioma tissues). (B) TUSC3 expression levels were quantified for comparison. A paired t-test was used to analyze the difference between TUSC 3 expression levels between glioma tissues and NAT. $\mathrm{P}=0.038$ vs. NAT. TUSC, tumor suppressor candidate; NAT, normal adjacent tissue.

and age between normal controls and patients with glioma were not statistically significant. However, when the patients with glioma were grouped according to median age, analyses revealed a significant increase in positive TUSC 3 expression in patients aged $\geq 43$ compared with patients $<43\left(\chi^{2}=8.531\right.$, $\mathrm{P}=0.007$; Table I).

Expression of TUSC3 in normal adjacent tissues and tumor tissues. The positive rates of TUSC 3 expression in normal adjacent tissue and glioma tissues were $76.9 \%$ (10/13) and $40.0 \%$ $(24 / 60)$, respectively. The difference in TUSC 3 expression levels between the two groups was revealed to be statistically significant $\left(\chi^{2}=5.854, \mathrm{P}=0.029\right.$; Table II). The results displayed

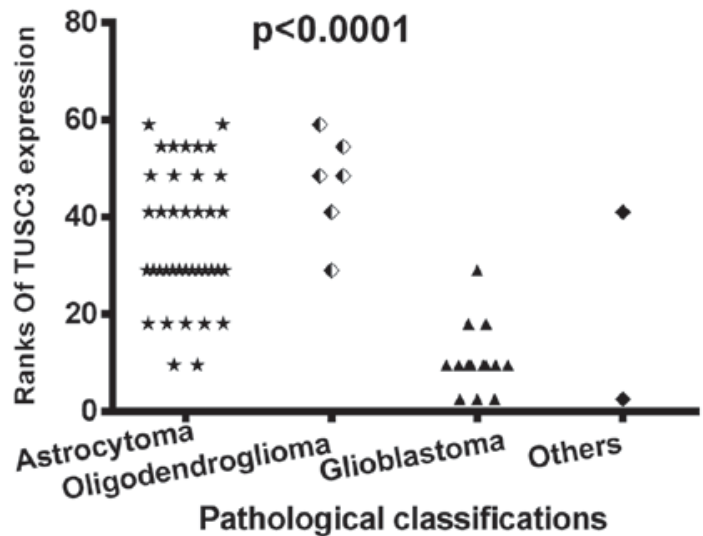

Figure 2. Expression of TUSC3 in association with pathological classification. TUSC 3 expression levels were compared between different pathological groups in patients with glioma. Whether there was a difference among the 4 groups was analyzed using the Wilcoxon rank sum test. $\mathrm{P}<0.05$. TUSC, tumor suppressor candidate.

in Fig. 1 demonstrating lower TUSC 3 expression levels in glioma tissues support this data.

Association between TUSC3 expression and tumor pathological classification. When the patients were grouped according to tumor pathological classification, which consisted of astrocytoma, oligodendroglioma, glioblastoma and other types (including one medulloblastoma and one anaplastic ependymoma), there was a statistically significant difference in TUSC 3 expression amongst the different pathological groups (sum rank, $\mathrm{P}<0.0001$; Fig. 2). Furthermore, the positive rates of TUSC 3 expression in the different pathological groups were compared using Fisher's exact test. Results revealed that the highest TUSC 3 expressionwas in the oligodendroglioma group (83.3\% positive rate), with little to no expression in glioblastoma or other types (Table I).

Expression of TUSC3 in association with the WHO grade of patients with glioma. The pathology of the tumor was evaluated, resulting in the categorization of the glioma tissues into grades. According to the WHO grade system, all of the glioma tissues were separated into grade I-IV (Fig. 3). As demonstrated in Fig. 3, TUSC 3 expression decreased as the WHO grade increased. As presented in Fig. 4, comparisons between the groups revealed that the TUSC 3 positive rate was significantly different amongst different WHO grades (sum rank, $\mathrm{P}<0.0001$; Fig. 4A). To further confirm the results above, a numerical value of correlation between TUSC 3 expression and the WHO 
A

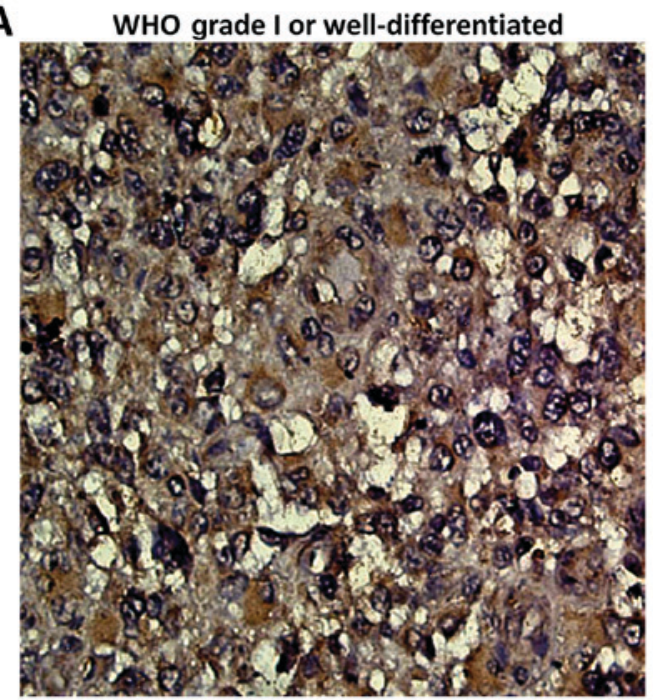

C WHO grade III or poorly differentiated

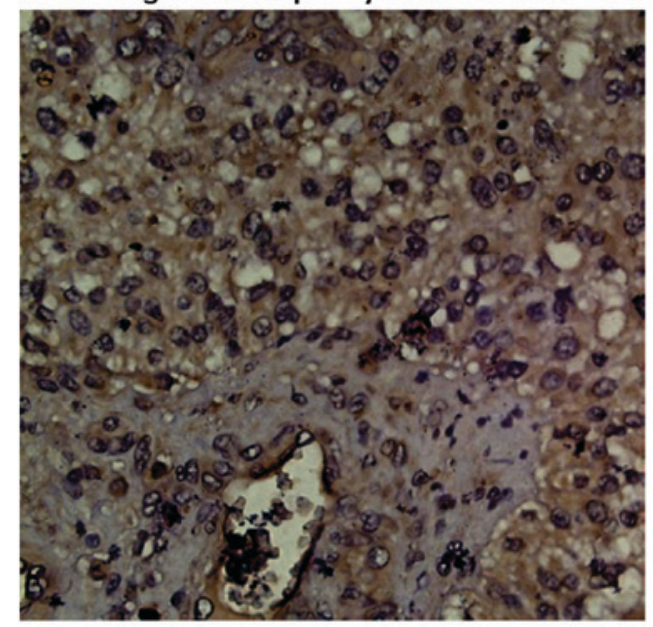

B WHO grade II or moderately-differentiated

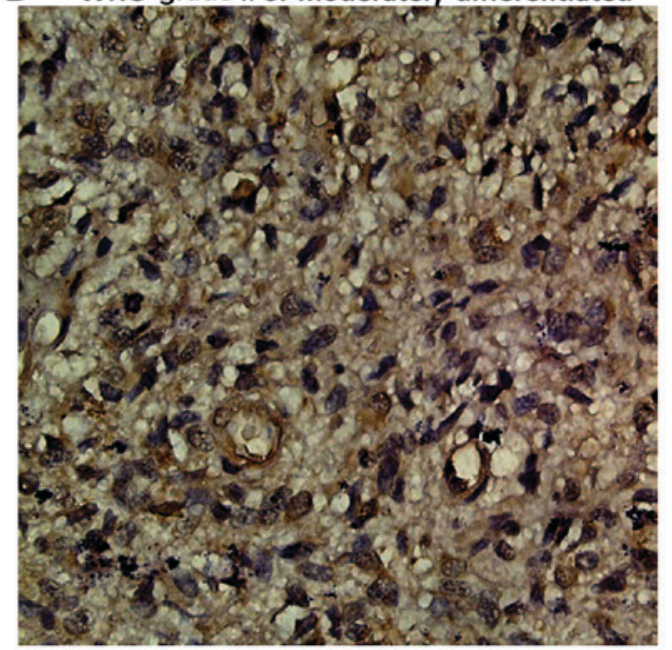

D WHO grade IV or undifferentiated

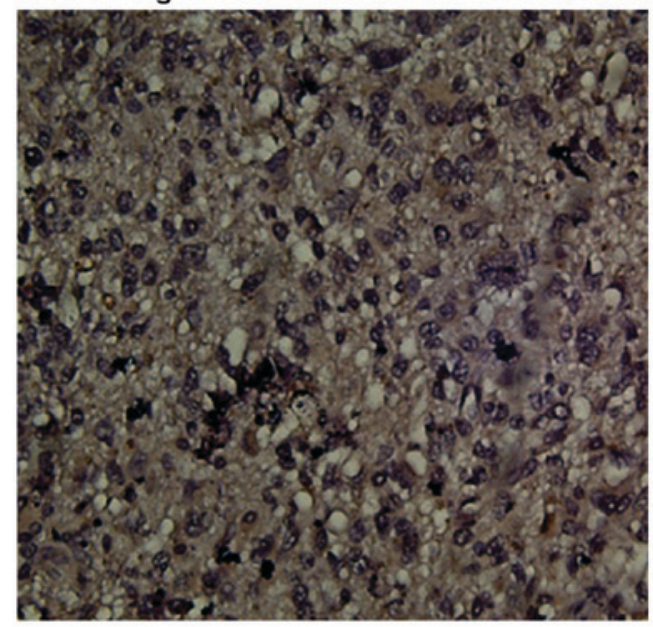

Figure 3. Immunohistochemical staining of tumor suppressor candidate 3 expression in human gliomas. (A) WHO Grade I or well-differentiated tumor tissue. (B) WHO Grade II or moderately-differentiated tumor tissue. (C) WHO Grade III or poorly differentiated tumor tissue. (D) WHO Grade IV or undifferentiated tumor tissue. Magnification x40. WHO, World Health Organization.
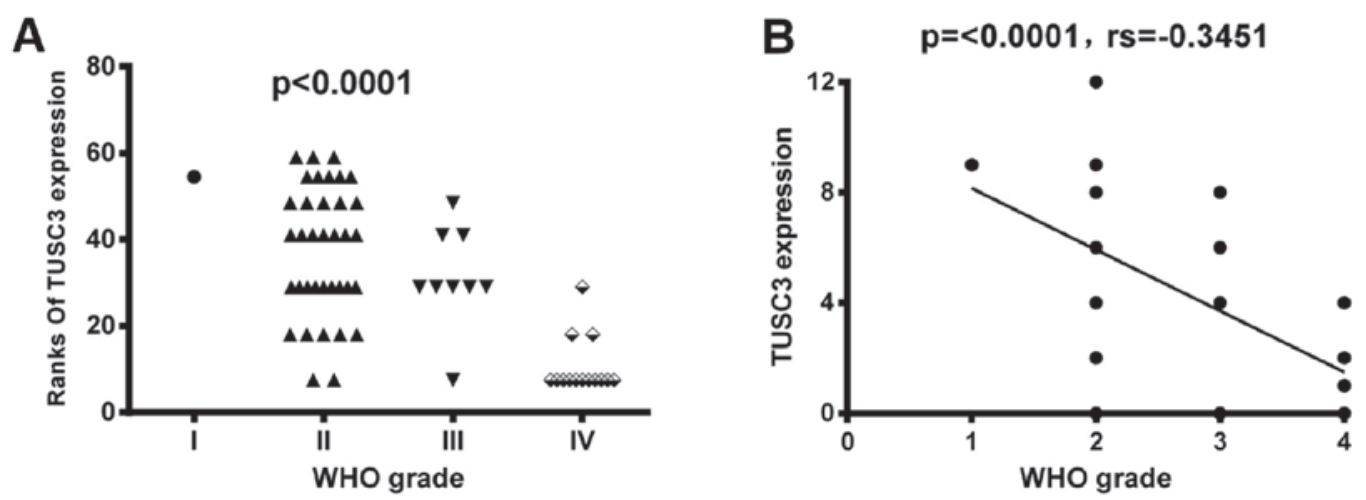

Figure 4. Expression of TUSC 3 in association with the WHO grade of patients with glioma. (A) TUSC3 expressions among different WHO grade classified patients with glioma were analyzed using a Wilcoxon's rank sum test. (B) Spearman's rank correlation coefficient method was used to examine the association between TUSC 3 expression and the WHO grade, $\mathrm{P}<0.05$. TUSC, tumor suppressor candidate; WHO, World Health Organization.

grade was determined using the Spearman correlation method. TUSC 3 expression demonstrated a negative correlation with WHO grade $(\mathrm{P}<0.0001$; rs=-0.3451; Fig. 4B). Thus, patients with higher grade tumors had lower TUSC3 expression levels.

\section{Discussion}

Clinical treatment results have demonstrated that gliomas are the main cause of mortality in patients with brain tumors, 
particularly in child patients with high-grade glioma (21). This reveals the urgency to identify unique indicators for diagnosis and prognosis, and to develop novel therapeutic approaches for gliomas.

The TUSC 3 gene is 224,265 bp in length, with 11 exons spanning the full length of the genomic DNA on chromosome 8p22 (7). TUSC3is considered an ortholog of the yeast Ost $3 p$, initially identified as a $34 \mathrm{kD}$ subunit in the yeast OST complex $(22,23)$. Human TUSC3 protein sequences share $\sim 20 \%$ identities of the protein domain organization with the yeast subunit OST3. Additionally, the two contain an active binding domain at the amino terminal, central acidic and zinc-binding domains, and a transmembrane domain at the carboxyl terminal (11). The transmembrane segments and N-terminal signal peptide are conserved (24).

TUSC3 serves notable functions in maintaining normal cell function. In 2008, Garshasbi et al (14) revealed TUSC3 homozygous deletion in 7 patients with non-syndromic ARMR in 4 sibships. Other studies additionally demonstrated that TUSC3 mutations or deletions are involved in the onset of ARMR $(15,25)$. Accumulated data has established that TUSC3 and cancer are notably associated (16-20). It has been previously demonstrated that TUSC3 is deleted or diminished in a number of cancer types, including prostate, ovarian, gastric, pancreatic, esophageal and lung cancer (16-20). Ribeiro et al (17) revealed that the loss of the TUSC3 gene has a potential association with the malignancy of oral squamous cell carcinoma. It was reported that TUSC3 loss is associated with a high mortality rate in larynx and pharynx squamous cell carcinoma (16). Previous observations had demonstrated lower expression levels of TUSC3 in patients who were lymph node metastasis positive (LNM+) compared with patients who were lymph node metastasis negative (LNM-) (18). It was additionally discovered that decreased TUSC3 levels predict a poor prognosis of patients with esophageal squamous cell carcinoma (20). However, to the best of our knowledge, there is no known data on the significance of TUSC 3 expression in patients with brain glioma, and the present study is the first that analyzes the association between the TUSC3 expression levels and the clinical characteristics of gliomas.

Through the use of western blot and tissue microarray assays, it was identified that TUSC3 expression was significantly lower in glioma tissues compared with normal adjacent tissues. Furthermore, TUSC3 expression demonstrated a significant negative correlation with WHO grade in patients with glioma $(\mathrm{P}<0.0001 ; \mathrm{rs}=-0.3451)$. Due to the fact that glioma WHO grade is an indicator for tumor malignancy and prognosis, the results of the present study indicated an association between low TUSC3 expression with a poorly-differentiated tumor grade and higher malignancy. The present results have the potential to make a notable contribution to the field of medicine. By combining the present research and further research in the future, TUSC3 may become a novel grading tool that assists in evaluating tumor malignancies and consequently a therapeutic regimen may be used for patients with glioma.

However, when the patients were grouped according to median age, analyses revealed a significant increase in TUSC3 positive expression in the patients who were age 43 or above compared with the patients who were younger than age 43 $\left(\chi^{2}=8.531, P=0.007\right.$; Table I). These results need to be verified using a larger sample. Furthermore, due to the screening rules for patient inclusion, there was a relatively small number of samples included in the present study. As a result, the TUSC3 expression in I-IV WHO grades of all histological types were not analyzed separately. Larger sample sizes will be used in further research.

With the expanding research in this area, the mechanisms for the involvement of the TUSC3 gene in cancer development have been illustrated. Vaòhara et al (26) and Horak et al (27) demonstrated that the loss of theTUSC3gene affects $\mathrm{N}$-glycosylation events in the development of ovarian cancer and prostate cancer. However, the precise molecular mechanism concerning the involvement of TUSC3 in the development of cancer remains unclear. As mentioned previously, a number of studies have demonstrated that TUSC3 is an integral endoplasmic reticulum protein involved in $\mathrm{N}$-glycosylation $(17,26)$. In addition, one previous study indicated that $\mathrm{N}$-glycosylation abnormalities affect the phosphoinositide 3-kinase-protein kinase B pathway, thus affecting the growth of tumor cells (28). However, further studies need to be performed regarding the mechanisms of TUSC3 involvement in glioma development.

Prior to the present study, limited mechanisms for TUSC3 gene dysfunction in tumors had been identified. Detected in multiple cancer types, aberrant TUSC3 reductions were identified due to a mutation resulting in its deletion $(17,29)$ as well as its promoter $\mathrm{CpG}$ island methylation $(27,30)$. Further research exploring the causes of TUSC3 dysfunction in gliomas, including genetic and epigenetic alterations, will aid understanding of the mechanism of TUSC3-associated tumorigenesis.

Due to its functional significance in protein maturation and the development of multiple tumor types, it has been proposed that TUSC 3 has the potential to be a useful biomarker and a therapeutic target in the diagnosis and therapy of ovarian cancer, prostate cancer and oral squamous cell carcinoma, particularly for refractory gliomas. There are various potentials for the therapeutic targeting of the TUSC3 protein in a tumor. Drug discoveries to reinstate TUSC3 function in diseases will focus on avenues including recombinant Ad-TUSC3 gene therapy, targeting the TUSC3 interactive proteins, or targeting destabilized oncogenic TUSC 3 mutants through the design of mutant-specific TUSC3 rescue drugs. Although substantial knowledge was gained from the present study, numerous structural aspects of TUSC3 function have remained elusive, including details of TUSC3 interactive proteins and the specifics of TUSC3 mutations in diseases. To be a promising therapy, TUSC3 targeting therapy requires further exploration.

\section{Acknowledgements}

The present study was supported by the Medical and Health Science and Technology Development Plan of Shandong Province (grant nos. 2015WS0213 and 2017GSF18195), the Natural Science Foundation of Shandong Province (grant nos. ZR2011HQ010, ZR2015HM077, ZR2016HQ50 and ZR2015CL030), the Chinese Postdoctoral Science Foundation (grant no. 2016M602154) and the National Natural Science Foundation of China (grant nos. 30901712 and 81301868). 


\section{References}

1. Walsh KM, Ohgaki H and Wrensch MR: Epidemiology. Handb Clin Neurol 134: 3-18, 2016.

2. Louis DN, Ohgaki H, Wiestler OD and Cavenee WK: WHO Classification of Tumours of the Central Nervous System. IARC Press, Lyon, 2007.

3. Schwartzbaum JA, Fisher JL, Aldape KD and Wrensch M: Epidemiology and molecular pathology of glioma. Nat Clin Pract Neurol 2: 494-503, 2006.

4. Vigneswaran K, Neill S and Hadjipanayis CG: Beyond the World Health Organization grading of infiltrating gliomas: Advances in the molecular genetics of glioma classification. Ann Transl Med 3: 95, 2015

5. Wen PY and Kesari S: Malignant Gliomas in Adults. N Engl J Med 359: 492-507, 2008.

6. Reifenberger G, Wirsching HG, Knobbe-Thomsen CB and Weller $\mathrm{M}$ : Advances in the molecular genetics of gliomas-implications for classification and therapy. Nat Rev Clin Oncol 14: 434-452, 2017.

7. Yu X, Zhai C, Fan Y, Zhang J, Liang N, Liu F, Cao L, Wang J and Du J: TUSC3: A novel tumour suppressor gene and its functional implications. J Cell Mol Med 21: 1711-1718, 2017.

8. MacGrogan D, Levy A, Bova GS, Isaacs WB and Bookstein R: Structure and methylation-associated silencing of a gene within a homozygously deleted region of humanchromosome band $8 \mathrm{p} 22$. Genomics 35: 55-65, 1996.

9. Zhou H and Clapham DE: Mammalian MagT1 and TUSC3 are required for cellular magnesium uptake and vertebrate embryonic development. Proc Natl Acad Sci USA 106: 15750-15755, 2009.

10. Molinari F, Foulquier F, Tarpey PS, Morelle W, Boissel S, Teague J, Edkins S, Futreal PA, Stratton MR, Turner G, et al: Oligosaccharyltransferase subunit mutations in non-syndromic mental retardation. Am J Hum Genet 82: 1150-1157, 2008.

11. Mohorko E, Owen RL, Malojčić G, Brozzo MS, Aebi M and Glockshuber R: Structural Basis of substrate Specificity of human oligosaccharyl transferase subunit N33/TUSC3 and its role in regulating protein N-Glycosylation. Structure 22: 590-601, 2014.

12. Jamaluddin MF, Bailey UM, Tan NY, Stark AP and Schulz BL: Polypeptide binding specificities of Saccharomyces cerevisiae oligosaccharyl-transferase accessory proteins Ost3p and Ost6p. Protein Sci 20: 849-855, 2011.

13. Zhang MJ, Xing LX, Cui M, Yang X, Shi JG, Li J, Zhang KJ, Zheng ZJ, Zhang FC, Li JL and Gao XC: Association of TUSC3 gene polymorphisms with non-syndromic mental retardation based on nuclear families in the Qinba mountain area of China. Genet Mol Res 14: 5022-5030, 2015.

14. Garshasbi M, Hadavi V, Habibi H, Kahrizi K, Kariminejad R, Behjati F, Tzschach A, Najmabadi H, Ropers HH and Kuss AW: A defect in the TUSC 3 gene is associated with autosomal recessive mental retardation. Am J Hum Genet 82: 1158-1164, 2008.

15. Garshasbi M, Kahrizi K, Hosseini M, Nouri Vahid L, Falah M, Hemmati S, Hu H, Tzschach A, Ropers HH, Najmabadi H and Kuss AW: A novel nonsense mutation in TUSC3 is responsible for non-syndromic autosomal recessive mental retardationin a consanguineous Iranian family. Am J Med Genet A 155A: 1976-1980, 2011.

16. Guervós MA, Marcos CA, Hermsen M, Nuño AS, Suárez C and Llorente JL: Deletions of N33, STK11 and TP53 are involved in the development of lymph node metastasis in larynx and pharynx carcinomas. Cell Oncol 29: 327-334, 2007.

17. Ribeiro IP, Marques F, Caramelo F, Pereira J, Patrício M, Prazeres H, Ferrão J, Julião MJ, Castelo-Branco M, de Melo JB, et al: Genetic gains and losses in oral squamous cell carcinoma: Impact on clinical management. Cell Oncol (Dordr) 37: 29-39, 2014
18. Yu X, Zhang K, Liu F, Zhang J, Zhai C, Cao L, Song X, Wang Y, $\mathrm{Li}$ B, Sun H and Du J: Tumor suppressor candidate 3 as a novel predictor for lymph node metastasis in lung cancer patients. Oncol Lett 12: 5099-5105, 2016.

19. Bashyam MD, Bair R, Kim YH, Wang P, Hernandez-Boussard T, Karikari CA, Tibshirani R, Maitra A and Pollack JR: Array-based comparative genomic Hybridization Identifies localized DNA amplifications and homozygous deletions in pancreatic cancer. Neoplasia 7: 556-562, 2005.

20. Yu X, Zhang J, Zhong H, Liu F, Liang N, Wang Y, Meng X and $\mathrm{Du}$ J: Decreased tumor suppressor candidate 3 predicts poor prognosis of patients with esophageal squamous cell carcinoma. Int J Med Sci 13: 963-969, 2016.

21. Rizzo D, Ruggiero A, Martini M, Rizzo V, Maurizi P and Riccardi R: Molecular biology in pediatric high-grade glioma: Impact on prognosis and treatment. Biomed Res Int 2015: 215135, 2015.

22. Das RC and Heath EC: Dolichyldiphosphoryloligosaccharideprotein oligosaccharyltransferase; solubilization, purification, and properties. Proc Natl Acad Sci USA 77: 3811-3815, 1980.

23. Kelleher DJ, Karaoglu D, Mandon EC and Gilmore R: Oligosaccharyl transferase isoforms that contain different catalytic STT3 subunits have distinct enzymatic properties. Mol Cell 12: 101-111, 2003.

24. Mohorko E, Glockshuber R and Aebi M: Oligosaccharyltransferase: The central enzyme of N-linked protein glycosylation. J Inherit Metab Dis 34: 869-878, 2011.

25. Khan MA, Rafiq MA, Noor A, Ali N, Ali G, Vincent JB and Ansar M: A novel deletion mutation in the TUSC3 gene in a consanguineous Pakistani family with autosomal recessive nonsyndromic intellectual disability. BMC Med Genet 12: 56 , 2011.

26. Vaòhara P, Horak P, Pils D, Anees M, Petz M, Gregor W, Zeillinger R and Krainer M: Loss of the oligosaccharyl transferase subunit TUSC3 promotes proliferation and migration of ovarian cancercells. Int J Oncol 42: 1383-1289, 2013

27. Horak P, Tomasich E, Vaňhara P, Kratochvílová K, Anees M, Marhold M, Lemberger CE, Gerschpacher M, Horvat R, Sibilia M, et al: TUSC3 loss alters the ER stress response and accelerates prostate cancer growth in vivo. Sci Rep 4: 3739, 2014.

28. Fang M, Shen Z, Huang S, Zhao L, Chen S, Mak TW and Wang X: The ER UDPase ENTPD5 promotes protein N-glycosylation, the Warburg effect, and proliferation in the PTEN pathway. Cell 143: 711-724, 2010.

29. Birnbaum DJ, Adélaïde J, Mamessier E, Finetti P, Lagarde A, Monges G, Viret F, Gonçalvès A, Turrini O, Delpero JR, et al: Genome profiling of pancreatic adenocarcinoma. Genes Chromosomes Cancer 50: 456-465, 2011.

30. Pils D, Horak P, Vanhara P, Anees M, Petz M, Alfanz A, Gugerell A, Wittinger M, Gleiss A, Auner V, et al: Methylation status of TUSC3 is a prognostic factor in ovarian cancer. Cancer 119: 946-954, 2013.

This work is licensed under a Creative Commons Attribution-NonCommercial-NoDerivatives 4.0 International (CC BY-NC-ND 4.0) License. 